in hanging-drop with $20 \%$ PEG8000 as precipitant. The typical crystal reaches $0.6 \times 0.3 \times 0.06 \mathrm{~mm}$ in two weeks. The crystals belong to the orthorhombic space group $\mathrm{P}_{2} 2_{2} 2_{1}$ with unit cell dimensions of $a=46.1 \AA, b=86.9 \AA$ and $c=126.5 \AA$. Native clatasets, with diffraction limit of $2.2 \AA$, at both room temperature and low temperature, have been collected on a Rigaku it-axis. Molecular replacement using the MAP kinase ERK2 as a model structure and multiple isonorphous replacement have been applied to solve the structure.

PS04.10.26 $1.7 \AA$ A STRUCTURE OF THE C-TERMINAL SH2 DOMAIN OF THE PS5 SUBUNTT OF HUMAN PHOSPHATD YLINOSITOL 3-KINASE. Simon Weston, Dean Derbyshire, Alex Breeze \& Richard Pauput, Zeneca Pharmaceuticals, Alderley Park, Macclesfield, UK

The C-terminal SH2 domain of the $85 \mathrm{kDa}$ regulatory subunit of human phosphatidylinositol 3-kinase (an enzyme responsible for a key mitogenic signal invoked by a variety of growth factors) complexed with a specific high-affinity phosphorylated tyrosine pentapeptide sequence from the platelet-derived growth factor receptor has been crystallised in spacegroup C2 and diffracts to $1.7 \AA$.

All molecular replacement efforts using other SH2 structures as trial models had failed - the structure could only be solved once the NMR structure carried out in our laboratory had produced a sufficiently accurate trial model. This was probably because the crystals were densely packed and the molecular replacement signal suffered from interference from cross-vectors. The structure is currently being refined and will be presented and compared to other SH2 structures.

\section{PS04.10.27 CRYSTAL STRUCTURE OF HUMAN} INTERLEUKIN-10 AT $1.6 \AA$ RESOLUTION. Alexander Zdanov, Celine Schalk-Hihi, Alexander Wlodawer, Macromolecular Structure Laboratory, NCI-Frederick Cancer Research and Development Center, ABL-Basic Research and Development Center, ABL-Basic Research Program, Frederick, Maryland, 21702

Interleukin-10 (IL-10) is a cytokine that inhibits production of a number of regulatory factors. The molecule is a homodimer of two intepenetrating monomers ( 160 amino acid residues each), forming a V-shaped structure. Each half of the structure consists of six $\alpha$-helices. four originating from one monomer and two from the other. The overal topology of the IL-10 bears close resemblance to interferon $\gamma$.

The crystal structure of human interleukin-10 (IL-10) has been refined at $1.6 \AA$ resolution against X-ray diffraction data collected at $100 \mathrm{~K}$ with the use of synchrotron radiation. Although similar to the IL-10 structure determined previously at room temperature, the low-temperature structure contains, in addition, four Nterminal residues, three sulfate anions, and 175 extra water molecules. Whereas the main-chain conformation is preserved, about $30 \%$ of the side chains, most of them on the protein surface, assume different conformations, and the protein itself appears to contract slightly at low temperature.

A computer model of a complex of IL-10 with its two soluble domains of its receptor has been generated based on the topological similarity of $I L-10$ to interferon- $\gamma$. The contact region between the IL-10 and each receptor shows excellent complementarity of polar and hydrophobic interactions, suggesting that the model is generally correct.
PR04.10.28 COMBINED STUDY OR PURINERGIC TRANSDUCTION USING CRYSTALLOGRAPIC AND ELECTROPHYSIOLOGICAL TECHNIQUES V. A. Panchenko, F. Rodrigues-Pascuali", M. T. Miras-Portugal", O.A.Krishtal, A. A. Bogomoletz Institute of Physiology, Kiev, Ukraine, "Departamento de Bioquimica, Facultad de Veterinaria, Universidad Complutense Madrid, Spain.

The purinergic transduction in manmalian brain is not conpletely investigated by this monent. The aim of this study was to compare the space distribution of diadenosine polyphosphates binding sites in rat brain by the means of autoradiography with possible contribution of this compounds in synaptic transmission mechanisms studied electrophysiologicaly. The distribution of the diadenosine tetraphosphate $(\mathrm{Ap} 4 \mathrm{~A})$ high affinity binding sites has been studied in rat brain by autoradiographic method using $[3 \mathrm{H}]-\mathrm{Ap} 4 \mathrm{~A}$ as the ligand. Due to specific activity of the labelled ligand, all of the tissues binding experiments were carried out to $1 \mathrm{nM}$ concentration. The Ap4A binding sites were widespread but heterogeneously distributed throe the rat brain. The range of densities was from 3 to 15 fmol/mg tissue in grey matter areas, while white matter was devoid of specific binding, showing background levels. In electrophysiological study the action of Ap4A on potential-dependent Ca channels was studied. Ap4A was found to cause a potentiation of $\mathrm{N}$-type Cat+ channels by $21 \pm 6 \%(n=12)$. This potentiation was observed in hippocampal CA3 neurons but not in cerebellar Purkinje neurons. The pharmacology of such potentiation was typical for $\mathrm{N}$-channels properties i.e. it was blocked by 5 micromolar of w-Conotoxin but not 50 micromolar nimodipine. It was possible to compare the data obtained by these two techniques and make a correlation in space distribution and functional properties of Ap4A receptors. In conclusion, our results support the existence of Ap4A specific binding sites and their contribution into Ca-regulated synaptic mechanisms.

\section{Protein Carbohydrate Structures}

MS04.11.01 ATOMLC INTERACTIONS BETWEEN PROTEINS AND CARBOHYDRATES. F. A. Quiocho" N. K. Vyas", M. N. Vyas" J. C. Spurlino*, L. E. Rodseth"* and D. R. Bundlet. "Howard Hughes Medical Institute and Department of Biochemistry, Baylor College of Medicine Houston, Texas 77030 , USA and TDepartment of Chemistry, University of Alberta, Edmonton, Alberta TGG 2C6. Canada

Major features of the atomic interactions between proteins and carbohydrates that will be presented are based on the following refined structures: i) L-arabinose-binding protein (ABP) complexed with L-arabinose, D-galactose, D-fucose, and 4 deoxy and halide derivatives of $\mathrm{D}$-galactose; ii) $\mathrm{D}$-glucose/D-galactose-binding protein (GGBP) complexed with D-glucose and D-galactose; iii) maltodextrin-binding protein (MBP) complexed with maltose, maltotriose, maltotetraose and $B$-cyclodextrin; and iv) antibody against the lipolysaccharides $\mathrm{O}$-antigen of Shigella flexneri in the absence and presence of trisaccharide and pentasaccharide $O$-antigen segments. The first three proteins serve as initial receptors for bacterial active transport and chemotaxis. All proteins that bind carbohydrates can be distinguished into two groups or types depending primarily on the location of the binding site. Whereas the sites in proteins belonging to Group I (e.g., ABP, GGBP, lacl family of repressors) are buried and able to sequester the ligands, those of group II (e.g, immunoglobulins, lectins, etc.) are near the protein surface. Each group further possesses somewhat unique features. $\mathrm{MBP}$ has a binding site groove which exhibits properties of the two groups. Features of protein-carbohydrate interactions that will be discussed include i) hydrogen-bonding interactions, ii) van der Waals interactions, especially those arising from stacking interactions with aromatic residues, iii) role of water molecule, iv) structural plasticity, v) bound oligosaccharide confomations, vi) specificity, and vii) affinity. 